\title{
An enhanced PSO-DEFS based feature selection with biometric authentication for identification of diabetic retinopathy
}

\author{
Umarani Balakrishnan**, Krishnamurthi Venkatachalapathy* \\ and Girirajkumar S. Marimuthu ${ }^{\dagger}$ \\ *Department of ECE, Trichy Engineering College \\ Tiruchirappalli 621132, Tamil Nadu, India \\ ${ }^{\dagger}$ Department of ICE, Saranathan College of Engineering \\ Tiruchirappalli 620012, Tamil Nadu, India \\ ‡umabkv@gmail.com
}

Received 21 July 2015

Accepted 15 November 2015

Published 7 January 2016

\begin{abstract}
Recently, automatic diagnosis of diabetic retinopathy (DR) from the retinal image is the most significant research topic in the medical applications. Diabetic macular edema (DME) is the major reason for the loss of vision in patients suffering from DR. Early identification of the DR enables to prevent the vision loss and encourage diabetic control activities. Many techniques are developed to diagnose the DR. The major drawbacks of the existing techniques are low accuracy and high time complexity. To overcome these issues, this paper proposes an enhanced particle swarm optimization-differential evolution feature selection (PSO-DEFS) based feature selection approach with biometric authentication for the identification of DR. Initially, a hybrid median filter (HMF) is used for pre-processing the input images. Then, the pre-processed images are embedded with each other by using least significant bit (LSB) for authentication purpose. Simultaneously, the image features are extracted using convoluted local tetra pattern (CLTrP) and Tamura features. Feature selection is performed using PSO-DEFS and PSO-gravitational search algorithm (PSO-GSA) to reduce time complexity. Based on some performance metrics, the PSODEFS is chosen as a better choice for feature selection. The feature selection is performed based on the fitness value. A multi-relevance vector machine (M-RVM) is introduced to classify the 13 normal and 62 abnormal images among 75 images from 60 patients. Finally, the DR patients are further classified by M-RVM. The experimental results exhibit that the proposed approach achieves better accuracy, sensitivity, and specificity than the existing techniques.
\end{abstract}

Keywords: Diabetic retinopathy (DR); least significant bit (LSB); local tetra pattern (LTrP); optical coherence tomography (OCT); hybrid median filter (HMF); particle swarm optimization (PSO); differential evolution feature selection (DEFS).

\$Corresponding author.

This is an Open Access article published by World Scientific Publishing Company. It is distributed under the terms of the Creative Commons Attribution 4.0 (CC-BY) License. Further distribution of this work is permitted, provided the original work is properly cited. 


\section{Introduction}

Diabetic Retinopathy (DR) is a major problem to the people affected by diabetic macular edema (DME). DR affects the people with high sugar or glucose level in the blood over a persistent period. High level of blood sugar may destroy the blood vessels in the posterior part of the eye. Therefore, the blurring occurs in the middle of the central visual field. The World Health Organization (WHO) recent survey space that there are 135 millions of people having diabetes and this number may increase up to 300 million by 2025. Our approach utilizes digital images such as optical coherence tomography (OCT) and retinal images to provide a better method for diagnosing the DR and identify the disease severity with security.

In general, the DR is classified into different types. Some of the major types are nonproliferative DR (NPDR) and proliferative DR (PDR). The NPDR is the initial stage of the DR in which the blood vessels leak the fluids and distort the eyesight. The PDR is an advanced stage of the DR, which creates greatest risk of visual loss than the NPDR. In that way, the PDR affects the following:

- Vitreous hemorrhage

- Traction retinal detachment (TRD)

- Neovascular glaucoma

The OCT image is a noninvasive diagnostic technique that extracts cross-sectional view of the retinal image. According to a recent survey, the diabetes is recognized as a main cause of blindness. If it is not diagnosed early and not treated in time, it leads to severe damage to the retinal structure that leads to partial or complete blindness. Figure 1 illustrates the diagnosis of DR.

Over a period of last decade, a lot of researches are conducted by various experts for automatic detection of DR based on the extraction of retinal and OCT images features. In this proposed work, the retinal image of the patient is embedded with the OCT image of the same patient to authenticate the patient for security. The incidence of the DME over 10-year period is described as follows:

- $20.1 \%$ in the younger - onset group.

- $25.4 \%$ in the oldest-onset group taking insulin.

- $13.9 \%$ in the oldest - onset without taking insulin.

Various existing approaches have several issues such as low accuracy, time complexity, and computational complexity. Enhanced image processing techniques are developed to overcome the issues of the existing methodology. The major contribution of this proposed work are defined as follows:

- Initially, pre-processing is performed using a hybrid median filter (HMF) to eliminate the noises from both OCT and retinal images of the patient.

- The pre-processed OCT image is embedded with the own retinal image to provide authentication. After the authentication, the images are extracted to get individual OCT and retinal images.

- Both image features are extracted by using convoluted local tetra pattern (CLTrP) along with the Tamura features.

- A hybrid feature selection strategy is used that incorporates with the particle swarm optimizationgravitational search algorithm (PSO-GSA) and PSO-Differential evolution feature selection (PSO-DEFS) techniques to reduce the time complexity.

- A M-RVM classifier is used to classify the normal and abnormal images that denote the affected and not affected DR. The abnormal images are further used to classify the disease through M-RVM.
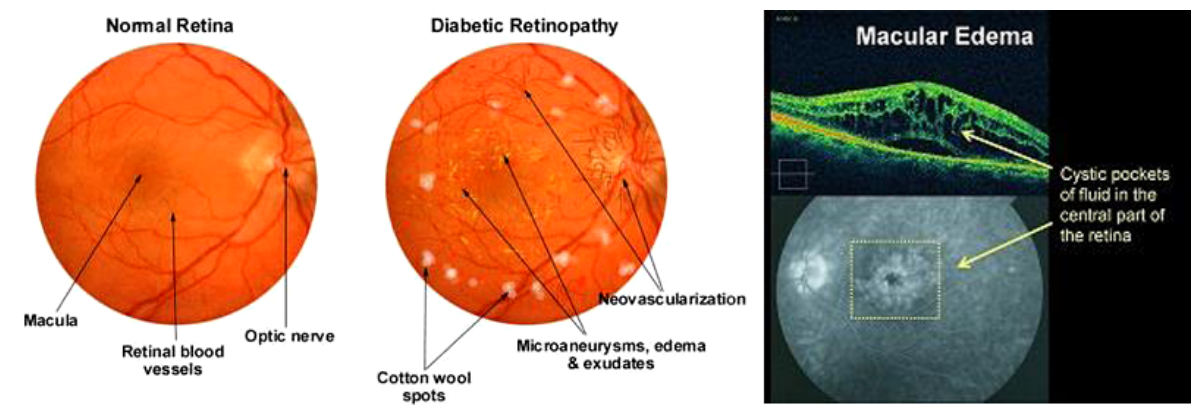

Fig. 1. An illustration for diagnosis of DR. 
The rest of the paper is structured as follows. Section 2 briefly explains the overview of the related works in the secure DR detection technique. Section 3 includes the detailed description of the proposed work including HMF, image embedding process, feature extraction, feature selection, and M-RVM classification. Section 4 describes the implementation details. Section 5 summarizes with a brief conclusive remark and discussion on future works.

\section{Related Works}

This section provides a comprehensive review of early detection of DR from OCT and retinal images. Singh and Tripathi proposed an automated DR diagnosis system to detect various lesions of the retina that were exudates, microaneurysms, and hemorrhages. A retinal grading algorithm was used for assessment of the severity of the disease. Therefore, the patients were referred to the corresponding specialist to provide better treatment. ${ }^{1}$ Sikorski et al. introduced an OCT to perform crosssectional imaging of the retina. This OCT successfully acted as an objective monitoring approach of the macular thickening before and after therapy. It also used to recognize the anatomy of DME and intra-retinal damage. ${ }^{2}$

Anitha et al. originated an automated system based on Artificial Neural Network (ANN) for eye disease classification. A back propagation neural network was used for the classification purpose to provide better performance results. ${ }^{3}$ Alireza et al. initiated a computational intelligence-based approach for the detection of exudates in DR images. But, the spatial relationship was not established between the detected exudates and fovea. And also the red lesions like hemorrhages and microaneurysms were not identified automatically. Matthew et al. proposed an automatic approach for learning variability models to identify excessive glycemic variability. An automatic feature selection was performed on a rich set of pattern recognition features. ${ }^{5}$

Lochan et al. developed an automated system to analyze the retinal images for extracting significant features of DR using image processing technique. The feature is captured from blood vessels, exudates, microaneurysms and texture. ${ }^{6}$ Gurudath et al. introduced an automatic identification of the presence of DR from color fundus images of the retina. This identification incorporates a three-layered ANN and support vector machine (SVM) to classify the retinal images. ${ }^{7}$

Srinivasan et al. utilized multi-scale histograms of oriented gradient descriptors. This histogram was considered as feature vectors of the SVM-based classifier. The spectral domain (SD)-OCT datasets are cross-validation from normal objects, agerelated macular degeneration, and DME. ${ }^{8}$ Saidha et al. detected Primary retinal pathology in multiple sclerosis using OCT. The functional corroboration of retinal dysfunction was provided via multifocal electroretinography in a subset of patients. ${ }^{9}$ Regatieri et al. examined choroidal thickness in patients with diabetes by SD OCT. This choroidal thickness was estimated from the posterior edge of the retinal pigment epithelium. ${ }^{10}$ Zhi et al. estimated modification occurred in early diabetes-induced in a TypeII diabetic mouse model using OCT and optical microangiography. ${ }^{11}$

Rodrigues et al. investigated the retinal modification prior to microangiopathy in type 2 diabetes mellitus (DM) patients without DR and in type 2 DM patients with mild DR. An analysis of the retinal layers was accurately performed with the Cirrus High Definition-OCT Review Software 6.0. ${ }^{12}$ Bressler et al. estimated the reproductivity of the central subfield thickness and volume measurement from OCT images with Zeiss status and Optovue RTVue. This measurement was converted into Equivalent Zeiss Stratus Metrics. ${ }^{13}$ Miura et al. measured the three-dimensional (3D) architecture of neovascularization in PDR by Doppler OCT (DOCT). The Prototype DOCT was used to measure the $3 \mathrm{D}$ vascular architecture at vitreoretinal adhesions. ${ }^{14}$

Meuer et al. determined the presence of Epiretinal Membranes (ERMs) and further retinal lesions using standardized grading of muscular (SD) OCT scans. It also described that the prevalence and interrelationships of ERMs. ${ }^{15}$ Gerendas et al. measured choroidal thickness on SD-OCT images by automated algorithms. Here, the choroidal pathology was correlated with retinal modification attributable to DME. ${ }^{16}$ Abhishek et al. designed an algorithm for accurate segmentation of intra-retinal layers for normal subjects and also patients with edema. This segmentation process was less prone to noise, and the pre-processing step was considered as an optional case. Therefore, noises were obtained in some of the segmentation process. ${ }^{17}$ 
Hasegawa et al. reviewed the medical records of 59 eyes with resolved macular edema, which is associated with branch retinal vein occlusion. During the presence or absence of the track lines, the eyes were classified in the SD-OCT images. It was obtained after the resolution of macular edema. ${ }^{18}$ Hunter et al. introduced the utilization of ultra-high resolution OCT subclinical anatomic alterations. It describes the suboptimum vision despite pseudophakic cystoid macula edema resolution. ${ }^{19}$ Zhang et al. proposed e-ophtha EX that is manually integrated with contoured exudates. The normalization, and denoising and detecting reflections and artifacts were performed in this proposed approach. ${ }^{20}$

\section{Proposed Work}

This section discusses the detailed description of the enhanced approach for identification of affected DR from OCT and retinal image. Figure 2 illustrates the overall working flow of enhanced feature extraction and selection for detection of DR. The major steps involved in this proposed methodology are:

- Pre-processing

- Image embedding

- Feature extraction

- Feature selection

- Classification

\subsection{Pre-processing}

Image pre-processing is an initial stage for identification of affected DR. Different techniques are used to remove the unwanted noise in the image. Two types of images such as OCT and retina are used to detect the type of diseases in DR. In this proposed work, a HMF is used to remove the noise in both images. The operation performed by HMF is discussed below:

\section{(1) Hybrid Median Filter}

The HMF is a windowed filter of nonlinear class that easily removes the noise while preserving the edges. It preserves the edge effectively than a square kernel median filter. The procedure for HMF is discussed as follows:

\section{HMF Algorithm}

1. Locate a cross window over element;

2. Pick up the elements;

3. Order the elements;

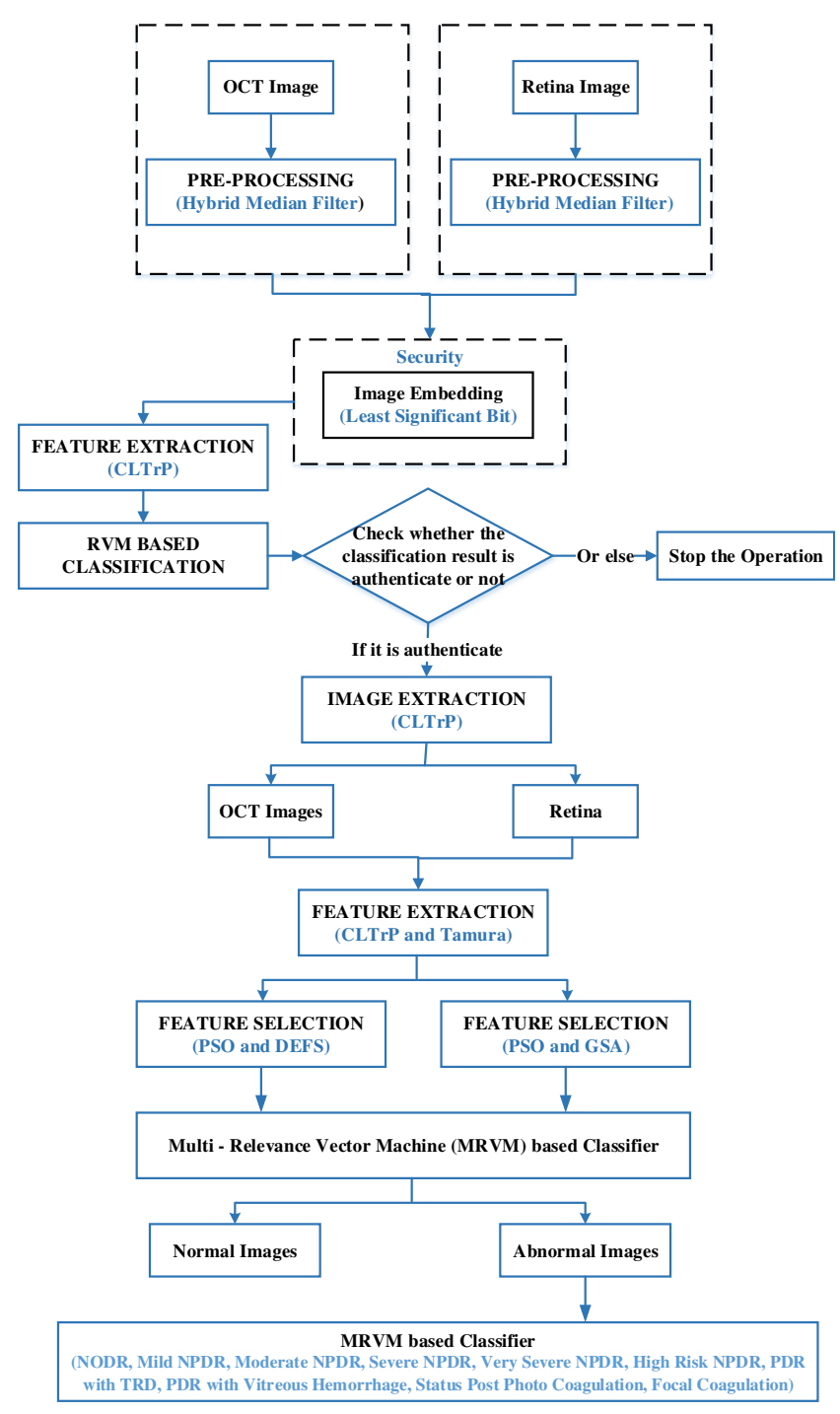

Fig. 2. An overall flow diagram of the proposed integrated approach for PSO-DEFS and PSO-GSA based feature selection for detection of DR.

4. Yield the middle element;

5. Locate a window over element;

6. Pick up the elements;

7. Order elements;

8. Yield the middle element;

9. Get the result from point 4 and 8 , and element itself;

10. Order elements;

11. Yield the middle element.

Based on this algorithm, the working flow of the HMF is described as follows: apply the Median Filter (MF) with a cross mask, apply MF with the $x$ mask, and return the median of obtained result and 


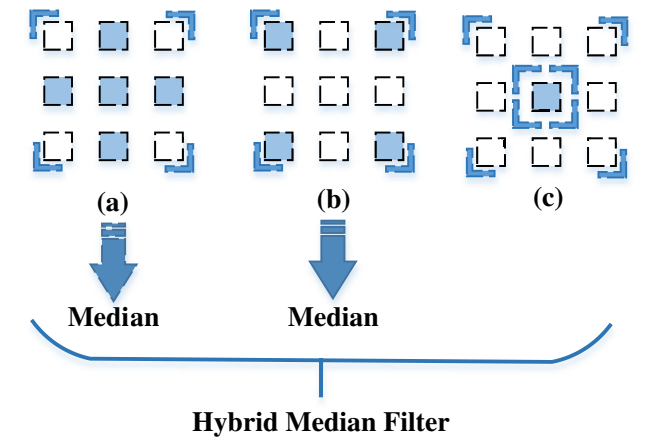

Fig. 3. (a) MF with cross mask, (b) MF with $x$ mask and (c) Median of $M_{P}$ and $M_{D}$.

element itself. The above mentioned workflow of HMF is depicted below in Fig. 3.

In Fig. 3, $M_{P}$ and $M_{D}$ denote the MF with cross mask and MF with the $x$ mask, respectively. This HMF has better corner preserving characteristics, which is widely used to improve the quality of the OCT and retinal images. Even with repeated application, the HMF does not excessively smooth image details and provides superior visual quality in the filtered image.

\subsection{Image embedding for security}

This image embedding is mainly used to provide authentication to the diabetic patient. In this paper, pre-processed OCT and retinal images of the diabetic patient are embedded with each other for security enhancement. The most widely used approach to embed the image is the usage of least significant bit (LSB). In general, the 8-bit image pixel is divided into most significant bit (MSB) and least significant bit (LSB) that is illustrated below:

\begin{tabular}{|c|c|c|c|c|c|c|c|}
\hline 7 & 6 & 5 & 4 & 3 & 2 & 1 & 0 \\
\hline & \multirow{2}{*}{\multicolumn{3}{|c|}{$\begin{array}{l}{ }_{\mathrm{MS}} \\
\end{array}$}} & \multirow{2}{*}{\multicolumn{4}{|c|}{$\begin{array}{c}1 \\
\text { LSB }\end{array}$}} \\
\hline & & & & & & & \\
\hline
\end{tabular}

The LSB represents the lowest significant bit in the byte value of the image pixel. In general, a conventional LSB requires 8 bytes of the pixel to store the 1 byte of secret image. But, in proposed LSB, the last 4 bits are used to embed the two images. Figure 4 depicts the step-by-step procedure for image embedding using LSB that are described as follows:

(1) Choose a cover image i.e., OCT image of size $\mathrm{M}^{*} \mathrm{~N}$ as an input.

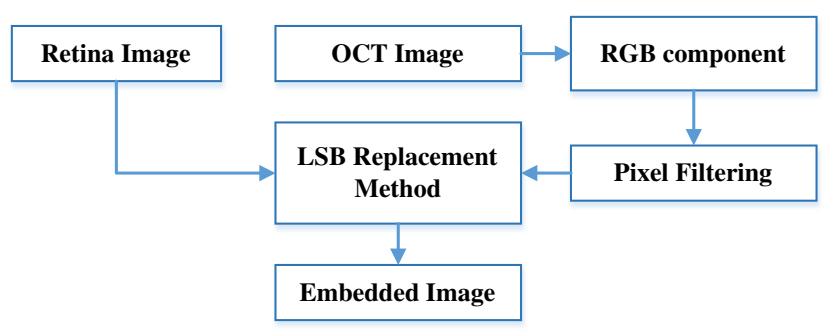

Fig. 4. Algorithm of LSB.

(2) The retinal image to be hidden is embedded in red, green and blue (RGB) component only of an image.

(3) Utilize a pixel selection filter to obtain the best areas to hide the information in the cover image to attain better rate. This filter is applied to LSB of each image pixel to hide the retinal image, leaving MSB.

(4) Finally, the image is hidden by using bit replacement method.

The features of the embedded image are extracted by using CLTrP. The extracted features are classified by using relevant vector machine (RVM) classifier. The obtained classification result is used to authenticate whether the embedded images match with the OCT and retinal images in the database. It is performed to check whether the embedded images belongs to the same patient or not. If the authentication is false, the operation should stop without any delay or else further processing is performed. This process is mainly used to enhance the security to avoid the mismatch of the OCT and retinal images of the patient.

After the authentication, embedded images are extracted by using the CLTrP approach. Again the OCT and retinal images are separated from each other to identify the disease in DR.

\subsection{Feature extraction}

The features of both OCT and retinal images are extracted by using CLTrP and Tamura features. A set of features is defined to represent the information for analysis and classification.

\section{(1) $C L \operatorname{TrP}$ approach}

The relationship between the center pixel and the neighbors is encoded by using the CLTrP technique. A Gabor transform is applied to obtain the convoluted value. A two-dimensional (2D) Gabor function is a Gaussian modulated by a complex 
sinusoid. It is specified in terms of frequency of the sinusoid $\varphi$ and the Standard Deviations (SDs), where $\sigma_{a}$ and $\sigma_{b}$ denote the SD of ' $a$ ' and ' $b$ ', respectively. The Gaussian envelope $(\tau)$ of the SDs is described as follows:

$$
\tau(a, b)=\frac{1}{2 \pi \sigma_{a} \sigma_{b}} e^{\left[-\left(\frac{1}{2}\right)\left(\frac{a 2}{\left.\sigma_{a}^{2}+\frac{b 2}{\sigma_{b}^{2}}\right)+2 \pi j \varphi a}\right]\right.} .
$$

The Gabor wavelets are obtained by dilation and rotation of the generating function $\tau(a, b)$ as follows:

$$
\tau_{l m}(a, b)=c^{-l} \tau\left(a^{\prime}, b^{\prime}\right)
$$

where $a^{\prime}$ and $b^{\prime}$ are estimated as follows:

$$
\begin{aligned}
a^{\prime} & =c^{-l}(x \cos \theta+y \sin \theta), \\
b^{\prime} & =c^{-l}(-x \sin \theta+y \cos \theta) .
\end{aligned}
$$

Here, $\theta=n \pi / J, \quad l \in\{0,1, \ldots, R-1\}$, and $m \in$ $\{0,1, \ldots, J-1\}$. ' $J$ ' and ' $R$ ' represents the number of desired orientation and scales, respectively. The response of the Gabor filter is the convolution of Gabor window with the image $(I)$, where $I=8$. Here, eight different orientation values for CLTrP are obtained, where each image contains 3328 features. This mean value of this orientation is estimated to obtain the specified result.

\section{(2) Tamura features}

In this proposed work, six general Tamura features such as coarseness, contrast, directionality, linelikeness, regularity, and roughness are extracted from both OCT and retinal images in the database. These features characterize the low-level statistical properties of the images. The properties and computation of the Tamura features are discussed as follows:

\section{(a) Coarseness $\left(E_{\text {crs }}\right)$}

It refers to the size and number of image primitives for analyzing the image. This value is used as the scale factor during extraction of other image features. This coarse feature consists of a small number of large primitives and a fine feature contains a large number of small primitives. This feature is estimated as follows:

$$
E_{\mathrm{crs}}=\frac{1}{n^{2}} \sum_{a}^{n} \sum_{b}^{n} 2^{j} p(a, b)
$$

where $n * n$ represents the image size, $p(a b)$ is a sum of each pixel, and $j$ enhances the difference of moving averages $\sum_{a} \sum_{b} p(a, b) / 2^{2 j}$, dominated a $2^{j} * 2^{j}$ neighborhood, along the horizontal and vertical directions.

(b) Contrast $\left(E_{\text {con }}\right)$

It defines the image quality in the narrow sense and also refers the intensity difference between the adjacent pixels. The image with high contrast has large intensity difference and vice versa. It is measured as follows:

$$
E_{\text {con }}=\frac{\sigma}{\left(\frac{\rho_{4}}{\sigma^{4}}\right)^{1 / 4}},
$$

where $\sigma$ denotes the SD of the image, and $\rho_{4}$ represents the fourth movement of the image.

\section{(c) Directionality $\left(E_{\text {dir }}\right)$}

It refers the shape of the image primitives and the placement rule, and also it consists of various recognition orientation. This feature is estimated as follows:

$$
E_{\mathrm{dir}}=1-n_{f} \cdot n_{p} \cdot \sum_{p}^{n_{p}} \sum_{\alpha \in r_{p}}\left(\alpha-\alpha_{p}\right)^{2} \cdot H_{\mathrm{LD}}(\alpha),
$$

where $H_{\mathrm{LD}}$ is local direction histogram, $n_{p}$ represents the number of peaks of $H_{\mathrm{LD}}, \alpha_{p}$ denotes $p$ th peak position of $H_{\mathrm{LD}}, r_{p}$ is the range of $p$ th peak between valleys, $n_{f}$ is the normalizing factor, and $\alpha$ denotes the quantized direction code.

(d) Line-likeness $\left(E_{\text {lin }}\right)$

It refers only the shape of the image primitives and is simultaneously directional. It is computed as follows:

$$
\begin{aligned}
E_{\operatorname{lin}}= & \sum_{a}^{n} \sum_{b}^{n} P_{D d}(a, b) \cos [(a-b) \\
& \times \frac{2 \pi}{n} / \sum_{a}^{n} \sum_{b}^{n} P_{D d}(a, b),
\end{aligned}
$$

where $P_{D d}(a, b)$ represents the $n * n$ co-occurrence matrix of points at distance $d$.

\section{(e) Regularity $\left(E_{\mathrm{reg}}\right)$}

It refers the variations of the texture primitive placement. A regular image consists of identical or similar primitives, and irregular image consists of multiple primitives. It is estimated as follows:

$$
E_{\mathrm{reg}}=1-n_{f}\left(\sigma_{\mathrm{crs}}+\sigma_{\mathrm{con}}+\sigma_{\mathrm{dir}}+\sigma_{\mathrm{lin}}\right),
$$

where $\sigma_{x x x}$ represents the SD of $E_{x x x}$. 
(f) Roughness $\left(E_{\text {rgh }}\right)$

It refers the tactile variations of physical surface, and it contains angular primitives. This roughness is computed by using the following equation:

$$
E_{\mathrm{rgh}}=E_{\mathrm{crs}}+E_{\mathrm{con}} .
$$

Also, the features such as energy, entropy and auto-correlation are extracted in this proposed work. The computation of this additional Tamura features is described below:

\section{(g) Energy $\left(E_{n}\right)$}

It is the texture measures of gray scale image and is estimated by adding the absolute values in a local neighborhood.

$$
E_{n}=\sum_{a=1}^{m} \sum_{b=1}^{n}(a-b)^{2} P(a, b),
$$

where $a$ and $b$ represent elements in the Tamura features, $P(a, b)$ is a probability values for changes between gray level $m$ and $n$.

(h) Entropy $\left(E_{t}\right)$

It describes a large amount of information about the images. This entropy estimates the information loss, and measures the intensity distribution randomness.

$$
\operatorname{Entropy}\left(C_{e}\right)=-\sum_{a} \sum_{b} P_{d}[a, b] \ln P_{d}[a, b],
$$

where $P_{d}[a, b]$ is a probability value for the changes between the gray levels ' $m$ ' and ' $n$ ' at a distance $d$.

(i) Auto-correlation $\left(E_{\mathrm{AC}}\right)$

The auto-correlation analyzes the regularity and coarseness of texture. It is evaluated as:

$$
\begin{aligned}
E_{\mathrm{AC}}= & 1-n_{f}(\operatorname{kurtosis}(\operatorname{con})+\operatorname{kurtosis}(\operatorname{dir}) \\
& +\operatorname{kurtosis}(\operatorname{lin})+\operatorname{kurtosis}(\operatorname{rgh}))+E_{\mathrm{rgh}} .
\end{aligned}
$$

After extracting the above mentioned Tamura features, the results are displayed as in Table 1.

\subsection{Feature selection}

The feature selection is the process of selecting the subset of relevant features. The relevant features in the OCT and retinal images are selected by using both PSO-GSA and PSO-Differential Evolution Feature Selection (PSO-DEFS) techniques.

\section{(1) $P S O-G S A$}

The best way to combine the PSO with GSA is to ensure their independent performance in a serial
Table 1. The results of tamura features.

\begin{tabular}{lc}
\hline Tamura Features & Feature Values \\
\hline Coarseness & 36.5123 \\
Contrast & 56.6010 \\
Directionality & 75.1649 \\
Line-likeness & 0.0541 \\
Regularity & 1 \\
Roughness & 57.6010 \\
Energy & $-2.0516 \mathrm{e}+06$ \\
Entropy & $6.2796 \mathrm{e}+06$ \\
Auto-correlation & $6.2521 \mathrm{e}+07$ \\
\hline
\end{tabular}

mode. This PSO-GSA hybridization treats any one of the particles in the swarm as a particle PSO and GSA by applying a co-evolutionary technique. Hence, each particle in the PSO-GSA updates the position with the contributions of both PSO velocity and GSA acceleration. Two terms of velocity updating information in proposed PSO-GSA contain these contributions. PSO is a population-based meta-heuristic method that is motivated by the intelligent collective behaviors of birds, fish, ants or human beings. Each particle in the PSO moves to a new position in the problem space according to the new velocity and previous positions. The velocity and new position of the particle are updated according to the following equations:

$$
\begin{gathered}
v_{j}^{k}(i+1)=w(i) v_{j}^{k}(i)+d_{1} * r_{1} *\left(P \text { best }_{j}^{k}-x_{j}^{k}(i)\right) \\
+d_{2} * r_{2} *\left(G \text { best }_{j}^{k}-x_{j}^{k}(i)\right) \\
x_{j}^{k}(i+1)=x_{j}^{k}(i+1)+v_{j}^{k}(i+1) \\
w(i)=\operatorname{rand} \times \frac{i}{i_{\max }} \times\left(w_{\max }-w_{\min }\right)+w_{\min }
\end{gathered}
$$

where $v_{j}^{k}(i)$ is the velocity of the particle ' $j$ ' in the $k$ th dimension at the $i$ th iteration and $x_{j}^{k}(i)$ is the current position of the particle. $d_{1}$ and $d_{2}$ are the acceleration coefficients that control the influence of Pbest and Gbest during the search process. $r_{1}$ and $r_{2}$ are the random numbers that lie within the range $[0,1]$. Pbest ${ }_{j}^{k}$ is the previous best position found by the particle ' $j$ ' and $G$ best $_{j}^{k}$ is the global best position among all the particles. $w(i)$ is the inertia weight. $w_{\max }$ and $w_{\min }$ are the maximum and minimum ranges of the inertia weight. ' $i$ ' is the current iteration and ' $i_{\max }$ ' is the maximum iteration. 
GSA is also a population-based meta-heuristic method inspired by the Newtonian physical law of gravitation and mass interactions. In GSA, each agent attracts each other agent through the gravity force that is directly proportional to the product of their masses and inversely proportional to the square of the distance between them. The velocity and the position of an agent at the next iteration $(i+1)$ are computed using the following equations:

$$
\begin{gathered}
v_{j}^{k}(i+1)=\operatorname{rand} \times v_{j}^{k}(i)+P_{j}^{k}(i), \\
x_{j}^{k}(i+1)=x_{j}^{k}(i)+v_{j}^{k}(i+1)
\end{gathered}
$$

\section{Procedure for PSO-GSA}

Input: Feature values, number of parameters to be optimized, and maximum number of iterations.

ALGORITHM: Search space identification $t=0$; Random initialization $X(t)$;

$$
\begin{aligned}
& \text { For } i=1: N \\
& \quad \text { Fitness evaluation of objects; } \\
& \text { val }=(\text { feaval }(i, j)-\text { feaval }(j, i)) \\
& \quad / \text { mean }(\text { each attribute }) ; \\
& \text { If val }>\text { mean } \quad(\text { attribute }) / 2 ;
\end{aligned}
$$$$
\text { Update the parameters of } G \text {, best, worst and } M \text {; }
$$$$
\text { End for }
$$

For $i=1: N$

Estimation of force on each attribute;

Estimation of acceleration and velocity of each object;

Update the position of agents by parameters to yield $X(t+1)$;

$t=t+1 ; / /$ until the stop criteria is reached

$$
\text { End if }
$$

End for

Get the best values;

Best values passed to DEFS; // attribute selected by $G S A$

For $i=1$ to size (inputs)

Initialize population;

Generate chromosome; // until number of iteration

Process single point cross over;

Each chromosome undergoes mutation with a fixed probability $\mu_{m}$;

Evaluate Fitness value using equation (19),

$$
\text { End for }
$$

The fitness value is evaluated using the following equation:

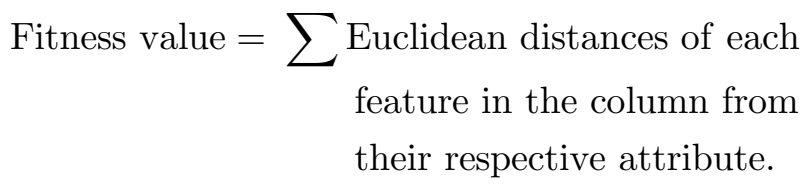

In this hybrid PSO-GSA, the PSO uses a memory ( $p$ best, $g$ best) to save the best solution, when the GSA uses the fitness value to adjust the accelerations. Here, the $g$ best is used to exploit the global best. This PSO-GSA solves a wide range of optimization problem.

(2) PSO-DEFS

In PSO, a particle denotes the each member of population i.e., swarms. Each particle has its individual best position that is denoted as $p$ best. After that, the optimal solution established by entire swarm is known as gbest. All the particles have its own fitness function that is defined by the optimization function. ${ }^{21}$ The below algorithm is the hybridization of PSO and DEFS.

PSO combined DEFS Algorithm

Inputs: Feature values, number of parameters to be optimized, maximum number of iterations

ALGORITHM: Randomly initialize position, number of iterations, and the velocity of each particle

While $i<$ maxiteration

Evaluate Fitness for each particle;

// Fitness

$\operatorname{Val}=(\operatorname{feaval}(i, j)-\operatorname{feaval}(j, i)) /$ skewness (each attribute);

If val > skewness (attribute) $/ 2$ pbest $=$ val;

For $i=1$ to PopulationSize do Update pbest of particle; Update gbest of particle;

For $i=1$ to PopulationSize do For $d=1$ to Dimensionality do Update the velocity of particle $i$; Update the position of particle $i$;

\section{End For}

End For

End For

End While

Return gbest; 
gbest values passed to DEFS // attribute selected by PSO
For $i=1$ to size (inputs)
Initialize population
Generate chromosome // until number of iteration

Process single point crossover

Each chromosome undergoes mutation with a fixed probability $\mu_{m}$;

Evaluate Fitness using equation (19)

End for

The PSO-GSA and PSO-DEFS approaches are used for the effective estimation of the fitness value. From the obtained result, the PSO-DEFS is the better choice for feature selection.

Figure 5 shows that if the number of generation is increased, then the fitness value is also gradually increased, when the experiment is iterated after it found the optimal solution. The experimental results show the result in terms of fitness value for ten generations. Here, the 10th generation delivers an optimum number of features with high fitness value. Based on this value, the features are selected from the extracted OCT and retinal images.

Based on the fitness value, the affected DR is identified from the OCT and retinal images. The feature selection process eliminates the insignificant features from the input space before the classification to reduce the time complexity issues. The dimensional reduction through the feature selection process reduces the classification time and

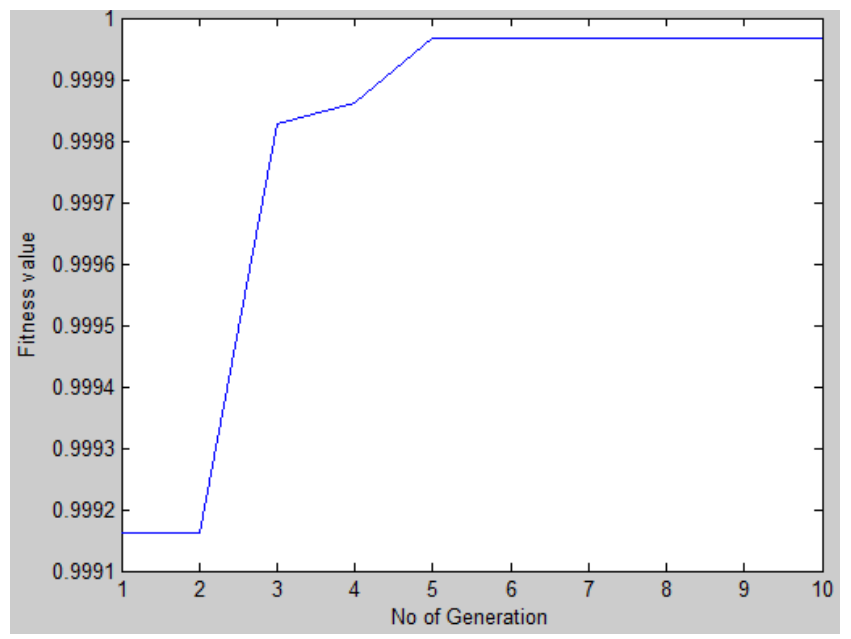

Fig. 5. Fitness estimation for PSO-DEFS. also improves the classification accuracy. This is possible due to the reduction in the overlap of different classes in the feature space. This feature selection mainly reduces the image dimension to reduce the computational cost. This results in the minimization of the misclassification of the images. The PSO-GSA and PSO-DEFS feature selection techniques provide the optimal separating hyperplane. This enables to obtain a clear decision boundary. Then, the selected features using PSOGSA and PSO-DEFS are classified for the detection of DR.

\subsection{Classification}

The selected features are classified by using M-RVM classification approach.

\section{(1) $M-R V M$}

The M-RVM becomes a popular choice for classification as it offers various merits over the SVM. This M-RVM is performed based on the Bayesian formulation of a linear model that is integrated with previous results. The obtained result is in a sparse representation with low computational cost. Let the training image collection $I_{T}=\left\{\left(I_{i}, c_{j}\right) \mid I_{i} \in D^{I_{i}}\right.$, $c_{j} \in(1,2, \ldots, n)$, where Image $I_{i}$ contains $m$ distinct feature terms, and $I_{i}=\left\{f_{1 i}, f_{2 i}, \ldots, f_{p i}\right\}$.

The multi-class classification on the selected image features is modeled as M-RVM by using below mentioned mathematical model:

$$
\begin{gathered}
c_{i}=I^{T} * \phi\left(I_{i}\right), \\
\left.c_{i}=\phi\left(I_{i}, I\right)+\delta_{k}\right) .
\end{gathered}
$$

Finally, the probabilistic classification equation of RVM based on the Gaussian prior distribution of the image features for $I_{i}$ from both OCT and retinal images is described as follows:

$$
P\left(\frac{c_{i}}{I_{i}}, \sigma^{2}\right)=\left(2 \pi \sigma^{2}\right)^{K / 2} \exp \left(-\frac{1}{2 \sigma^{2}} *\left[c_{i}-\phi\left(I_{i}\right)\right]^{2}\right) .
$$

This formula is used to estimate the prediction of the classes. The M-RVM prediction is more reliable than the SVM classifier. The M-RVM outputs probabilities of class membership than the point estimation. It delivers a conditional distribution in the class prediction to obtain the resultant abnormal images. The multi-class classification enables accurate classification of a set of images using more 


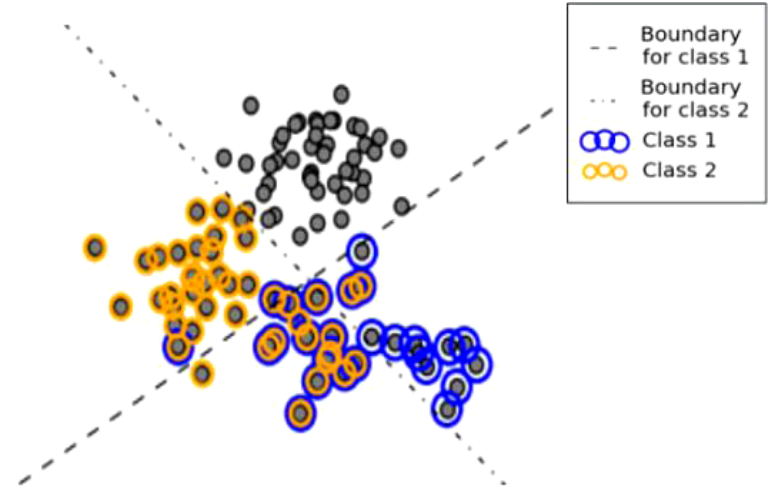

Fig. 6. Multi-class classification results.

than two classes. The multi-class classification ensures better interpretability and high computational efficiency. It is also possible to gain knowledge about the class by inspecting its corresponding classifier since each class is represented by one and one classifier only. Figure 6 shows the multi-class classification results.

Based on the supervised learning process, the features and labels for the images are learnt and training of the images is performed. Then, the decision vector is determined based on the different

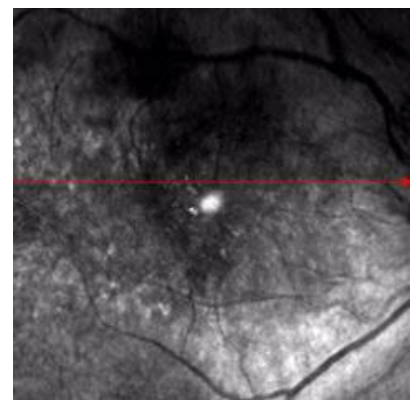

(a)

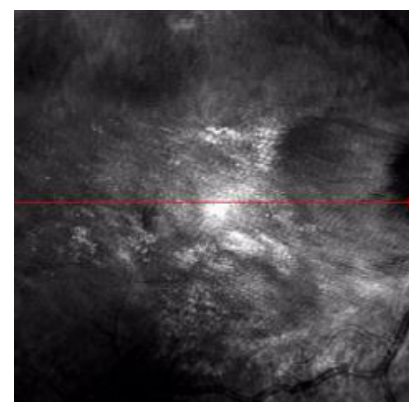

(e)

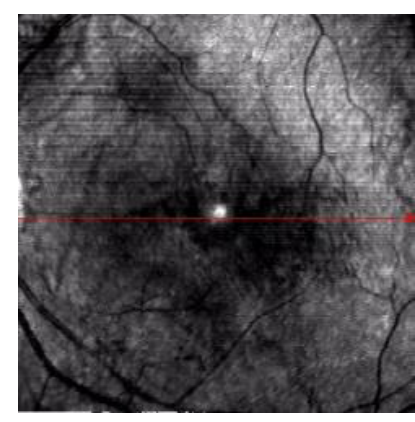

(b)

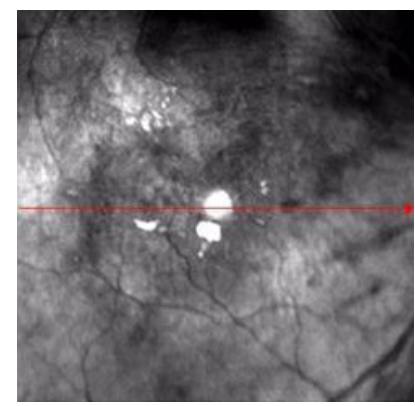

(f) class labels. Various stages of the diseases are classified based on the class labels. Here, CSME represents Clinically Significant Macular Edema. The affected DR is identified as any one of the following diseases:

- NODR

- Mild NPDR (with CSME/without CSME)

- Moderate NPDR (with CSME/without CSME)

- Severe NPDR (with CSME/without CSME)

- Very severe NPDR (with CSME/without CSME)

- High-risk NPDR (with CSME/without CSME)

- PDR with vitreous hemorrhage (with macular edema/without molecular edema)

- PDR with TRD

- Status post photo coagulation (stable/unstable)

- Focal coagulation (done/not done)

Figure 7(a) shows very severe NPDR with CSME, Fig. 7(b) shows moderate NPDR without CSME, Fig. 7(c) shows severe NPDR with CSME, Fig. 7(d) shows NODR, Fig. 7(e) shows PDR with Vitreous hemorrhage with macular edema, Fig. 7(f) shows PDR with CSME, Fig. 7(g) shows mild NPDR with CSME, and Fig. 7(h) shows PDR with TRD.

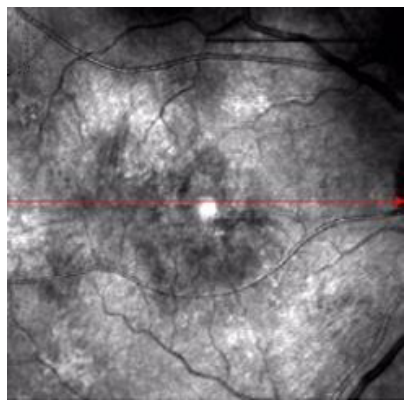

(c)

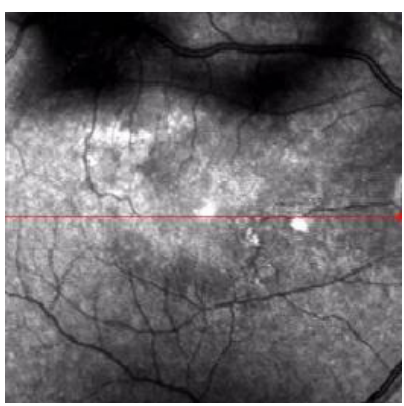

(g)

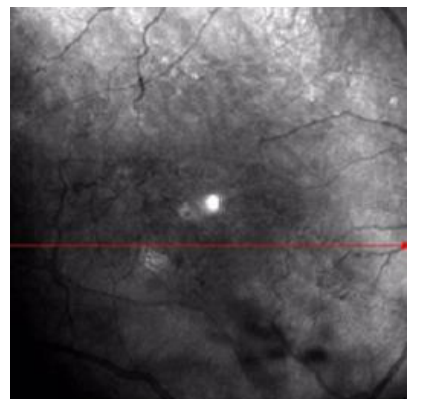

(d)

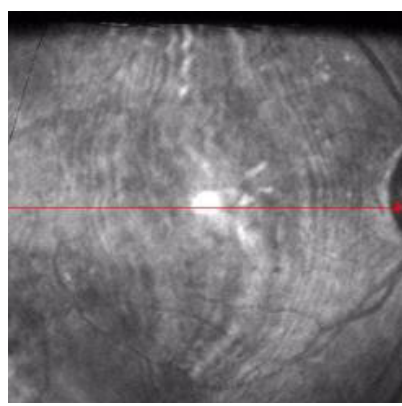

(h)

Fig. 7. (a) Very severe NPDR with CSME, (b) moderate NPDR without CSME, (c) severe NPDR with CSME, (d) NODR, (e) PDR with vitreous hemorrhage with macular edema, (f) PDR with CSME, (g) mild NPDR with CSME, and (h) PDR with TRD. 


\section{Performance Analysis}

This section presents the experimental results obtained from the investigation of the proposed work for the identification of DR from both OCT and retinal images. The evaluation metrics such as accuracy, sensitivity, and specificity are evaluated to estimate the performance of the proposed methodology. During the testing phase, 60 different samples are taken and tested accordingly.

Our research work provides major focus on the patients affected by DME. The total number of images collected for this research work are 75 diseased optical coherence tomography (OCT) images, 15 normal OCT images (out of 100 images) and diseased CSME, PDR, NPDR, and severe PDR images. These real-time datasets are collected from Mahatma Gandhi Eye Hospital, Trichy. To enhance the effectiveness of the proposed work, the image dataset of 100 labeled images, which includes 13 normal and 47 abnormal images from 60 patients are considered.

The input OCT/retinal image is collected from the dataset. The retinal image is captured by the fundus camera in the RGB form. The pre-processing is performed in both the OCT and the retinal image to filter the noise. After that, the pre-processed retinal image is embedded with the OCT image for authentication. This operation is clearly shown in Fig. 8.

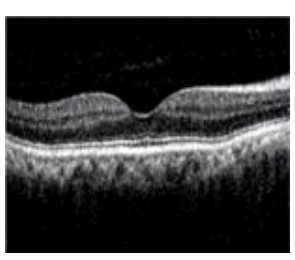

(a)

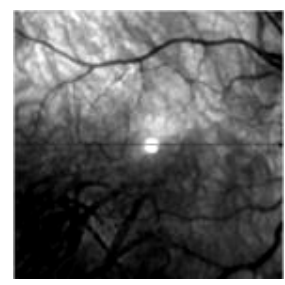

(d)

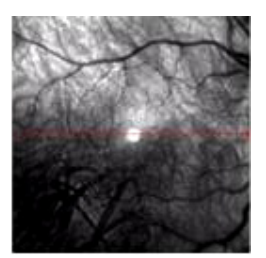

(b)

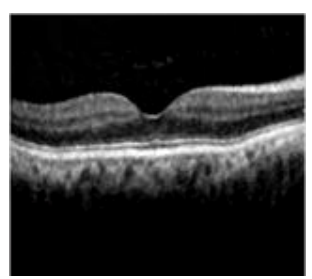

(e)
Fig. 8. (a) Original OCT image, (b) original retinal image, (c) pre-processed OCT image, (d) pre-processed retinal image, and (e) embedded image for authentication.

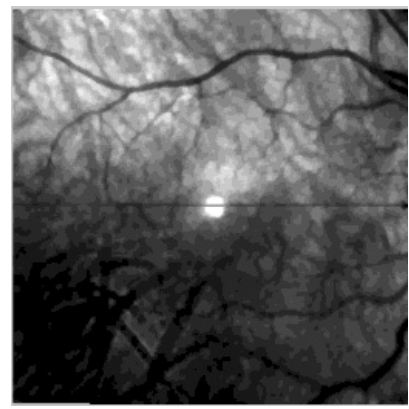

(a)

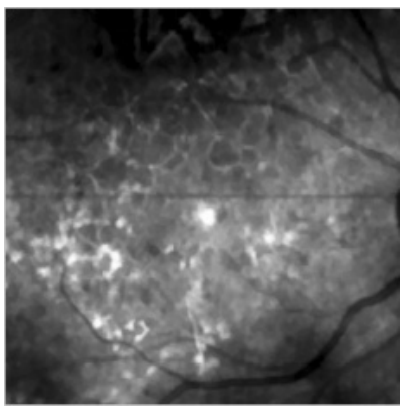

(b)
Fig. 9. (a) Extracted normal retinal image, and (b) extracted abnormal retinal image.

If the embedded image mismatches with the original image of the patient in the database, then the operation should stop or else further process is performed to detect the DR for authenticated patient. For that, the retinal image is extracted from the embedded image as shown in Fig. 9. If the extracted image is normal, then no issue or else the type of disease is identified by classifying the features.

The performance metrics such as accuracy, sensitivity, and specificity for the PSO-GSA and PSODEFS during feature selection are compared with the existing techniques such as probabilistic neural network (PNN) and multi-SVM (M-SVM). In this proposed work, the accuracy, sensitivity, and specificity are estimated for 3000 features.

The PSO-GSA shows better performance on high-dimensional functions. The convergence rate of the PSO-GSA is higher than the standard PSO and GSA. The PSO-GSA converges smoothly to the optimum value without any abrupt oscillations. This approach is highly successful in finding the best solution and obtaining a higher quality solution with better convergence property. The MRVM classifier requires a smaller amount of relevance vector than the SVM. The testing time, design complexity and cost of the M-RVM classifier are also lower than the SVM classifier. The MRVM classifier improves the classification accuracy of the medical images and reduces the training time. The decision function of the RVM classifier is much sparser than the SVM while maintaining its detection accuracy. This leads to the significant reduction in the computational complexity of the decision function, thereby making it more suitable for real-time applications. SVM requires tuning during the training phase, but RVM does not 
require tuning of a regularization parameter. The computational complexity of the RVM classifier is lower than the SVM classifier. The PNN classifier requires high memory requirements and representative training set. Moreover, PNN approach executes slowly in the real-time applications.

\subsection{Confusion matrix $-M-R V M$ classification}

This matrix is determined among the target class and output class. This confusion matrix exhibits the results of true positive (TP), true negative (TN), false positive (FP), and false negative $(\mathrm{FN})$. In this proposed work, the TP is $25, \mathrm{TN}$ is $39, \mathrm{FP}$ is 0 , and $\mathrm{FN}$ is 1 . The TP denotes the exact detection of the number of images, FP represents the incorrect detection of the number of images, $\mathrm{TN}$ is the exact rejection of the number of images, and $\mathrm{FN}$ denotes the incorrect rejection of the number of images. The green color represents TP and TN values. The red color denotes the FP and FN values. The confusion matrix exhibits the results of the TP rate, FP rate, accuracy, sensitivity, and specificity. The confusion matrix for M-RVM classification is clearly shown in Fig. 10.

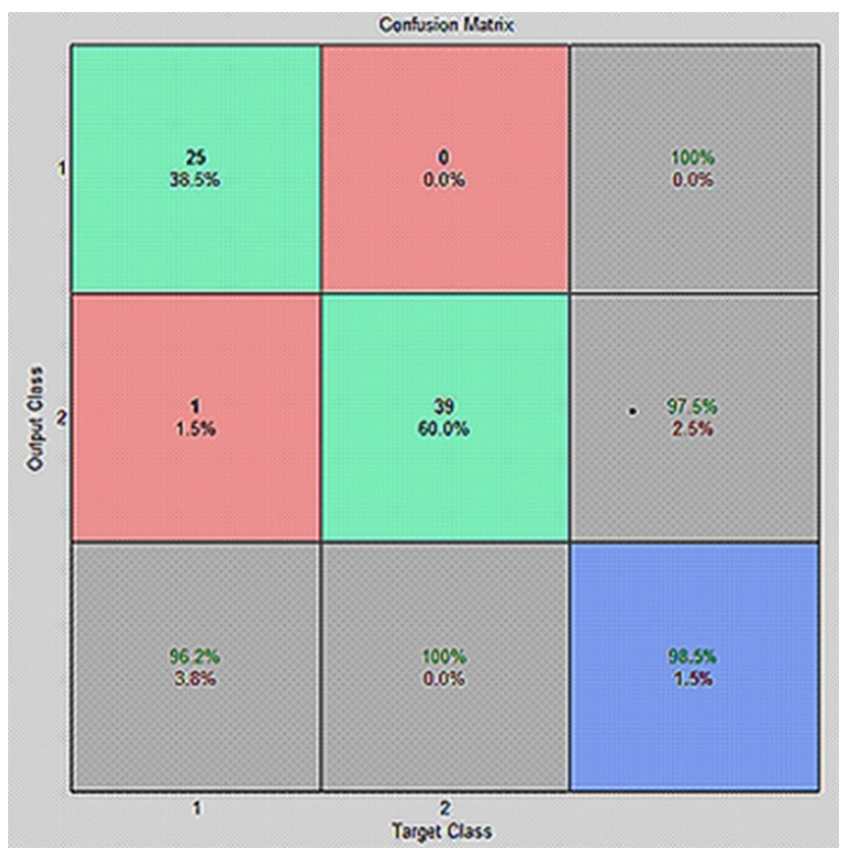

Fig. 10. Confusion matrix-M-RVM classification.

\subsection{Accuracy}

Accuracy defines the proximity of measurement results to the true value. Accuracy is estimated by using the following equation:

Accuracy $=\frac{\text { Number of } \mathrm{TP}+\text { Number of } \mathrm{TN}}{\text { Total population }}$,

where the total population is the summation of the number of TP, number of FP, the number of $\mathrm{FN}$, and number of TN.

Figure 11(a) depicts the accuracy of existing PNN and M-SVM and proposed M-RVM for feature

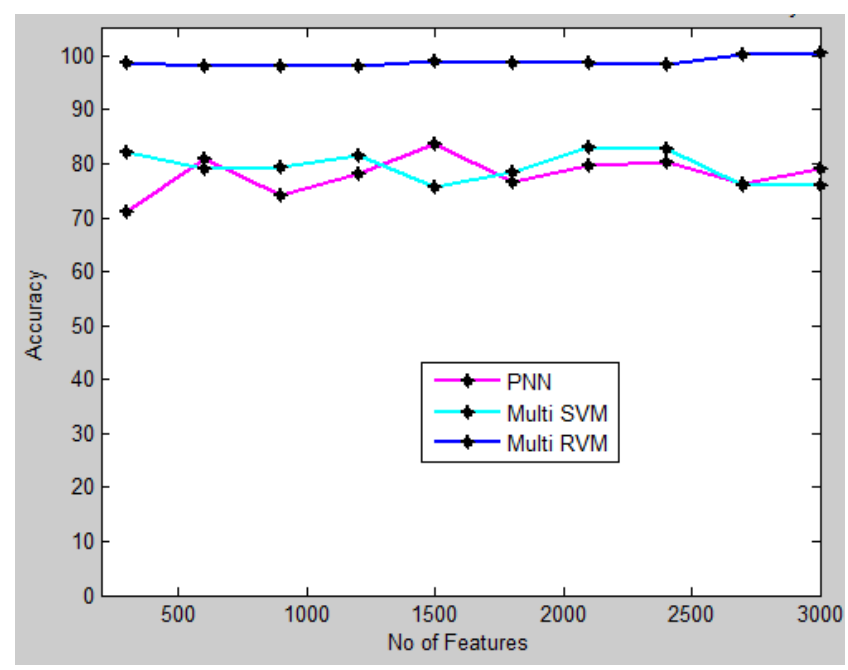

(a)

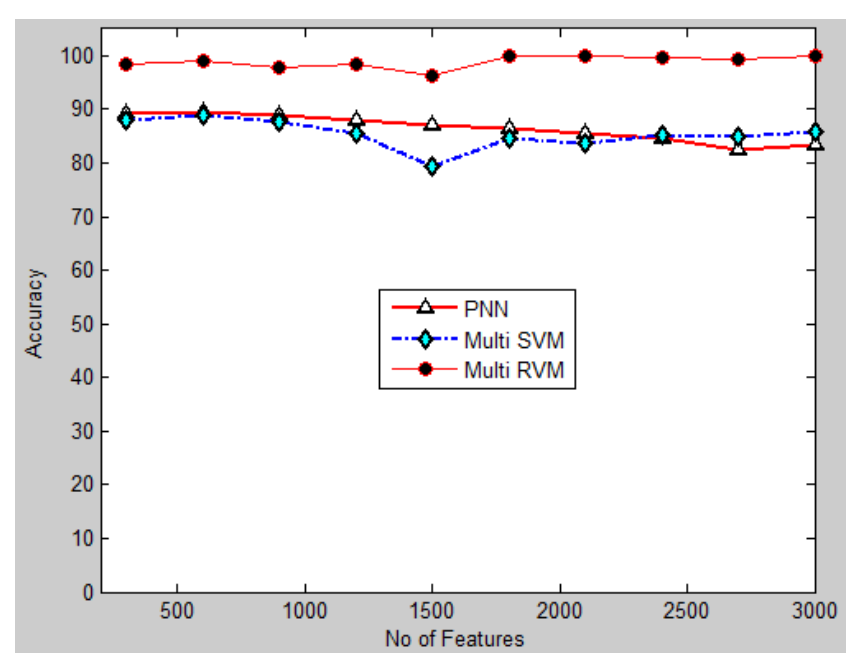

(b)

Fig. 11. (a) and (b) Accuracy analysis of M-RVM, PNN and Multi-SVM approaches. 


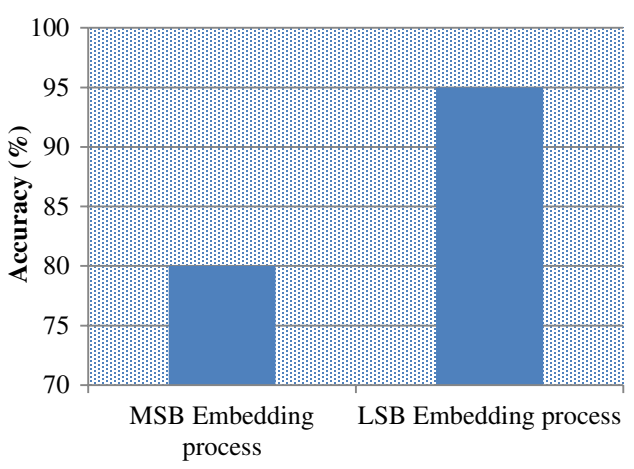

Fig. 12. Accuracy analysis of LSB and MSB embedding process.

selection using PSO-GSA. The accuracy of the proposed M-RVM is higher than the existing techniques. Similarly for feature selection using PSO-DEFS, the obtained accuracy result is shown in Fig. 9(b).

Figure 11(b) depicts that the accuracy for PSODEFS algorithm outperforms than the PSO-GSA algorithm. The accuracy of the classification result for both PSO-GSA and PSO-DEFS are $97.23 \%$ and $99.12 \%$, respectively. Therefore, the proposed PSODEFS has high accuracy, when compared with PSO-GSA.

The accuracy of the LSB embedding process is compared with the MSB embedding process. Figure 12 shows the comparison between the accuracy of the LSB and MSB embedding processes. The LSB embedding process achieves better performance than the MSB embedding process. Figure 13 shows the steganographic image obtained using MSB embedding process.

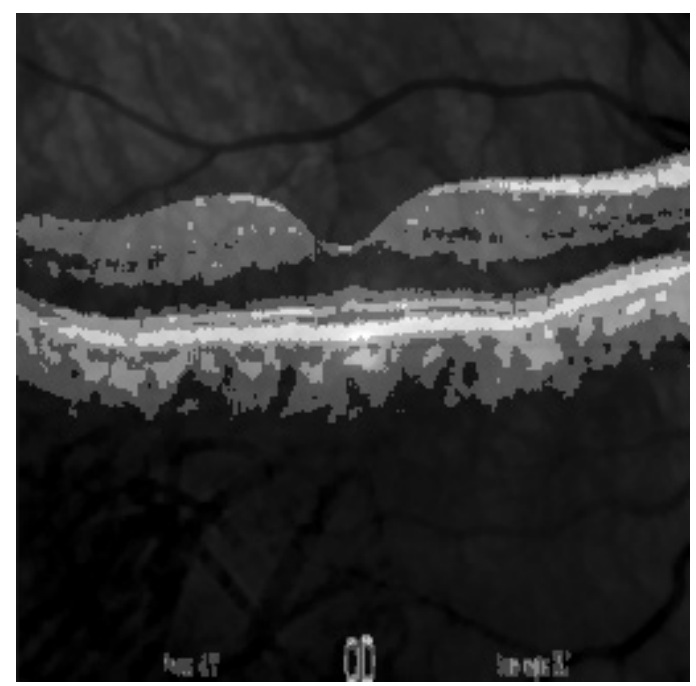

Fig. 13. Steganographic image obtained using MSB embedding process.

\subsection{Specificity}

Specificity rate is described as the probability that a test result will be negative once the DR is not present and is evaluated as follows:

$$
\text { Specificity }=\frac{\text { Number of TN }}{\text { Number of FP }+ \text { Number of TN }} \text {. }
$$

Figure 14(a) shows the specificity of the existing PNN and M-SVM techniques and proposed M-RVM classifier for PSO-DEFS.

Figure 14(b) depicts the specificity of the existing PNN and M-SVM techniques and proposed M-RVM classifier for PSO-GSA. Here, the PSO-DEFS

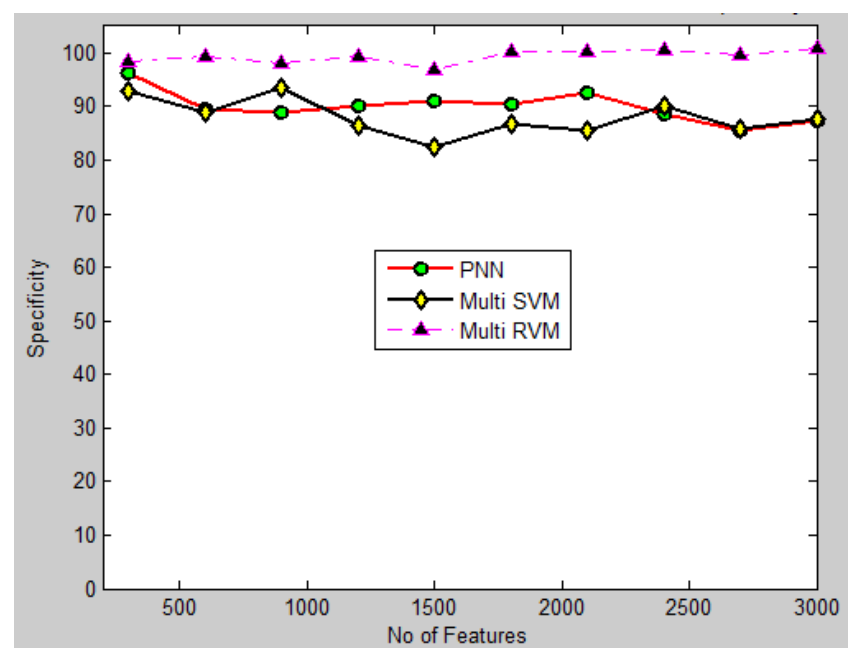

(a)

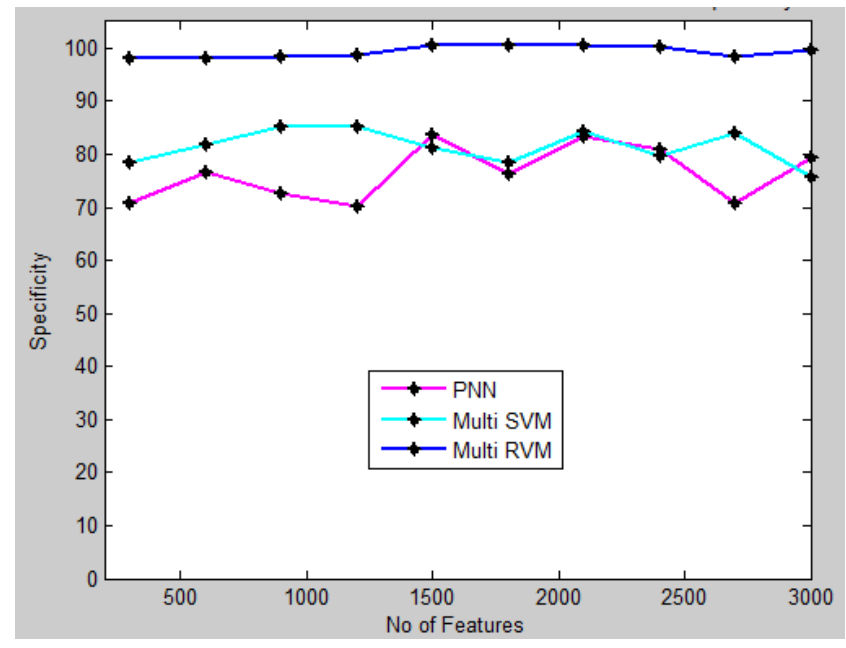

(b)

Fig. 14. (a) and (b) Comparative Analysis of specificity for M-RVM, PNN and Multi-SVM approaches. 


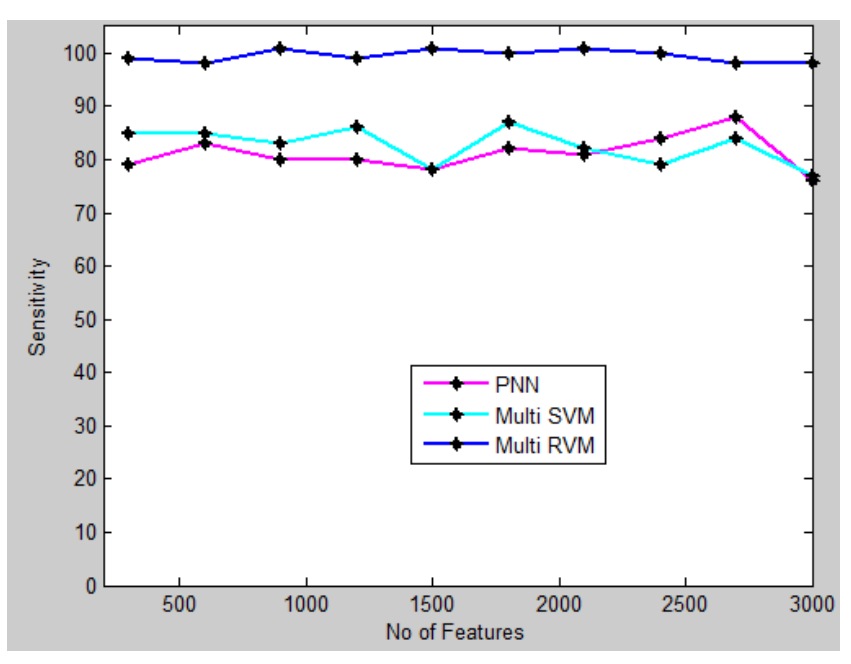

(a)

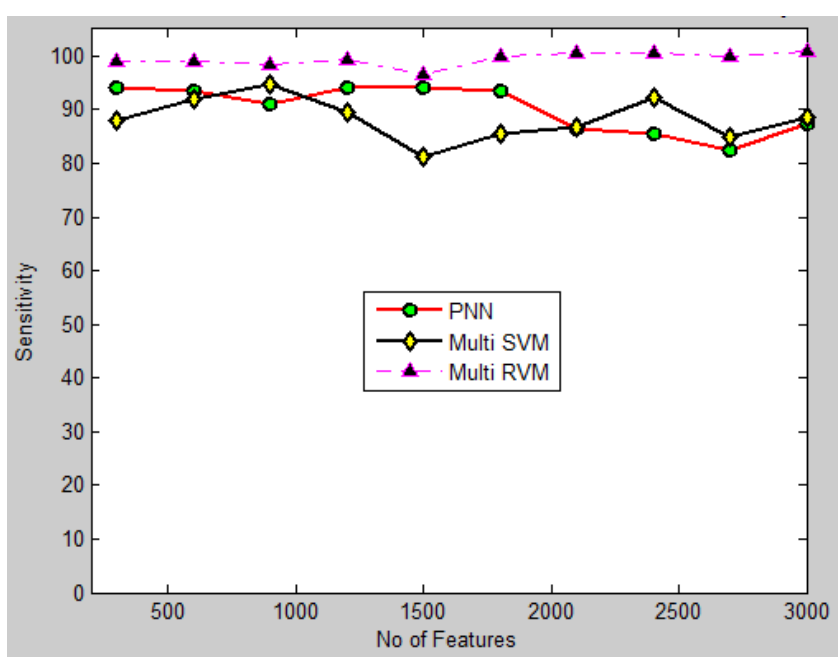

(b)

Fig. 15. (a) and (b) Sensitivity result of M-RVM, PNN and Multi-SVM approaches.

outperforms the PSO-GSA. Because, the specificity of the classification result for both PSO-GSA and PSO-DEFS are $90.28 \%$ and $98.7 \%$, respectively. Therefore, the proposed PSO-DEFS has high sensitivity, when compared with PSO-GSA.

\subsection{Sensitivity}

Sensitivity rate is defined as the probability that a test result is positive when the DR is present. It is evaluated as follows,

$$
\text { Sensitivity }=\frac{\text { Number of TP }}{\text { Number of TP }+ \text { Number of FN }} \text {. }
$$

According to this equation, the sensitivity of the proposed methodology is estimated. Figure 15(a) illustrates the sensitivity of the existing PNN and M-SVM techniques and proposed M-RVM classifier for PSO-GSA.

Figure 15(b) depicts the sensitivity of existing PNN and M-SVM and proposed M-RVM classification for PSO-DEFS algorithm. This PSO-DEFS outperforms than the PSO-GSA algorithm.

Here, the PSO-DEFS outperforms than the PSOGSA. Because the sensitivity of the classification results for both PSO-GSA and PSO-DEFS are 92.62\% and $98.2 \%$, respectively. Therefore, the proposed PSODEFS has high sensitivity when compared with PSOGSA. Finally, based on the class labels, the diseases are classified by using M-RVM. From this classification, we conclude that the patient is affected by Moderate NPDR without CSME disease in DR.

\section{Conclusion and Future Work}

This section presents the conclusion and future scope of the proposed work. In this paper, an enhanced PSO-DEFS based feature selection for DR identification with biometric authentication is proposed. At first, the input images are pre-processed by HMF. The pre-processed images are embedded with each other using LSB for authentication purpose to provide security. If the image is authenticated, then the further process is performed, or else the operation is stopped. Simultaneously, the image features are extracted using CLTrP and Tamura features for the authenticated image. The feature selection is performed by PSO-DEFS and PSO-GSA to reduce time complexity. Based on the fitness value, the feature selection is performed. The M-RVM classification approach is applied to classify the 13 normal and 62 abnormal images among 75 images from 60 patients. Then, the patients with DR are further classified by M-RVM. The obtained accuracy, sensitivity, and specificity are $99.12 \%, 98.2 \%$, and $98.7 \%$, respectively. Finally, we conclude that the experimental results show that the proposed work achieves better accuracy, sensitivity, and specificity than the existing M-SVM and PNN techniques. In future, the research work can be extended to detect glaucoma.

\section{References}

1. N. Singh, R. C. Tripathi, "Automated early detection of diabetic retinopathy using image analysis techniques," Int. J. Comput. Appl. 8, 18-23 (2010). 
2. B. L. Sikorski, G. Malukiewicz, J. Stafiej, H. Lesiewska-Junk and D. Raczynska, "The diagnostic function of OCT in diabetic maculopathy," Mediat. Inflamma. 2013, 1-12 (2013).

3. J. Anitha, D. Selvathi and D. J. Hemanth, "Neural computing based abnormality detection in retinal optical images," in IEEE Int. Advance Computing Conf. 2009, 630-635 (2009).

4. A. Osareh, B. Shadgar, R. Markham, "A computational-intelligence-based approach for detection of exudates in diabetic retinopathy images," IEEE Trans. Inf. Technol. Biomed. 13, 535-545 (2009).

5. M. Wiley, R. Bunescu, C. Marling, J. Shubrook, F. Schwartz, "Automatic detection of excessive glycemic variability for diabetes management," 10th Int. Conf. Machine Learning and Applications and Workshops (ICMLA), pp. 148-154 (2011).

6. K. Lochan, P. Sah, K. K. Sarma, "Innovative feature set for retinopathic analysis of diabetes and its detection," 3rd National Conf. Emerging Trends and Applications in Computer Science (NCETACS), pp. 240-245 (2012).

7. N. Gurudath, M. Celenk, H. B. Riley, "Machine learning identification of diabetic retinopathy from fundus images," Signal Processing in Medicine and Biology Symp. (SPMB), 2014 IEEE, pp. 1-7 (2014).

8. P. P. Srinivasan, L. A. Kim, P. S. Mettu, S. W. Cousins, G. M. Comer, J. A. Izatt et al., "Fully automated detection of diabetic macular edema and dry age-related macular degeneration from optical coherence tomography images," Biomed. Opt. Express 5, 3568-3577 (2014).

9. S. Saidha, S. B. Syc, M. A. Ibrahim, C. Eckstein, C. V. Warner, S. K. Farrell et al., "Primary retinal pathology in multiple sclerosis as detected by optical coherence tomography," Brain 134, awq346 (2011).

10. C. V. Regatieri, L. Branchini, J. Carmody, J. G. Fujimoto, J. S. Duker, "Choroidal thickness in patients with diabetic retinopathy analyzed by spectral-domain optical coherence tomography," Nat. Inst. Health Public Access 32, 563-568 (2012).

11. Z. Zhi, J. R. Chao, T. Wietecha, K. L. Hudkins, C. E. Alpers, R. K. Wang, "Noninvasive imaging of retinal morphology and microvasculature in obese mice using optical coherence tomography and optical microangiography," Invest. Ophthalmol. Vis. Sci. 55, 1024-1030 (2014).

12. E. B. Rodrigues, M. Urias, F. Penha, E. Badaró, R. Meirelles, E. Novais et al., "Early neural retinal changes detected by spectral-domain optical coherence tomography in type 2 diabetes mellitus," Invest. Ophthalmol. Vis. Sci. 55, 4427-4427 (2014).
13. S. B. Bressler, A. R. Edwards, C. M. Andreoli, P. A. Edwards, A. R. Glassman, G. J. Jaffe et al., "Reproducibility of optovue RTVue optical coherence tomography retinal thickness measurements and conversion to equivalent zeiss stratus metrics in diabetic macular edema," Transl. Vis. Sci. Technol. 4, 1-11 (2015).

14. M. Miura, Y.-J. Hong, Y. Yasuno, D. Muramatsu, T. Iwasaki, H. Goto, "Three-dimensional vascular imaging of proliferative diabetic retinopathy by Doppler optical coherence tomography," Am. J. Ophthalmol. 159, 528-538 (2015).

15. S. M. Meuer, C. E. Myers, B. E. Klein, M. K. Swift, Y. Huang, S. Gangaputra et al., "The epidemiology of vitreoretinal interface abnormalities as detected by spectral-domain optical coherence tomography: The beaver dam eye study," Ophthalmology 122, 787-795 (2015).

16. B. S. Gerendas, S. M. Waldstein, C. Simader, G. Deak, B. Hajnajeeb, L. Zhang et al., "Three-dimensional automated choroidal volume assessment on standard spectral-domain optical coherence tomography and correlation with the level of diabetic macular edema," Am. J. Ophthalmol. 158, 10391048 (2014).

17. A. M. Abhishek, T. T. Berendschot, S. V. Rao, S. Dabir, "Segmentation and analysis of retinal layers (ILM \& RPE) in optical coherence tomography images with edema," 2014 IEEE Conf. Biomedical Engineering and Sciences (IECBES), pp. 204-209 (2014).

18. T. Hasegawa, N. Masuda, N. Ogata, "Highly reflective line in optical coherence tomography images of eyes with macular edema associated with branch retinal vein occlusion," Am. J. Ophthalmol. 159, 925-933. e1 (2015).

19. A. A. Hunter, S. P. Modjtahedi, K. Long, R. Zawadzki, E. K. Chin, J. J. Caspar et al., "Improving visual outcomes by preserving outer retina morphology in eyes with resolved pseudophakic cystoid macular edema," J. Cataract Refract. Surg. 40, 626-631 (2014).

20. X. Zhang, G. Thibault, E. Decenciere, B. Marcotegui, B. Laÿ, R. Danno et al., "Exudate detection in color retinal images for mass screening of diabetic retinopathy," Med. Image Anal. 18, 1026-1043 (2014).

21. U. Balakrishnan, K. Venkatachalapathy, R. S. Giri, "A hybrid PSO-DEFS based feature selection for identification of diabetic retinopathy," Curr. Diabetes Rev. 11, 182-190 (2015). 